Check for updates

Cite this: Nanoscale Adv., 2019, 1, 937

\title{
Ball milling: a green technology for the preparation and functionalisation of nanocellulose derivatives
}

\author{
Carmen C. Piras, (D) a Susana Fernández-Prieto ${ }^{b}$ and Wim M. De Borggraeve (D) *a \\ Ball milling is a simple, fast, cost-effective green technology with enormous potential. One of the most \\ interesting applications of this technology in the field of cellulose is the preparation and the chemical \\ modification of cellulose nanocrystals and nanofibers. Although a number of studies have been reported \\ in the literature, the potential of this technique in the field of cellulose nanoparticles has not been fully \\ exploited. This minireview aims at putting existing work into perspective, highlighting the significance \\ and the potential of this green, sustainable technique to facilitate the identification of areas of future \\ development.
}

Received 24th September 2018 Accepted 29th December 2018

DOI: $10.1039 / c 8 n a 00238 j$

rsc.li/nanoscale-advances chemical actions. Nanofibers can be obtained by mechanical shearing actions such as high-pressure homogenization, cryocrushing, microfluidization, high intensity ultrasonic treatments and grinding.8 ${ }^{8,9}$ Chemical treatments (e.g. acid hydrolysis, TEMPO-mediated oxidation and enzymatic reactions) can facilitate this process as well as biological pretreatments (e.g. enzymatic degradation in the presence of microorganisms like fungi), which can help the removal of lignin and hemicellulose. ${ }^{6,10}$ The resulting fibrils can form highly viscous, entangled networks in aqueous media at concentrations below $1 \mathrm{wt} \%$. Cellulose nanofibers can also be produced in highly crystalline form by different types of bacteria (e.g. Komagataeibacter Xylinus)..$^{11,12}$ These are cultivated in aqueous nutrient media and the fibres are excreted at the interface with the air. Bacterial nanocelluloses (BNCs) have a high weight-average molecular weight and a good mechanical stability. The extraction of the crystalline regions contained within the cellulose microfibrils allows collecting crystalline rod-like particles called cellulose nanocrystals (CNCs). ${ }^{\mathbf{8}, 9}$ These particles have a well-defined shape and are mainly obtained by acid hydrolysis, which degrades the amorphous regions and disrupts the hydrogen bonds. Compared to CNFs, CNCs are highly crystalline and have a smaller aspect ratio (i.e. length to width ratio). CNFs, CNCs and BCNs are more broadly defined

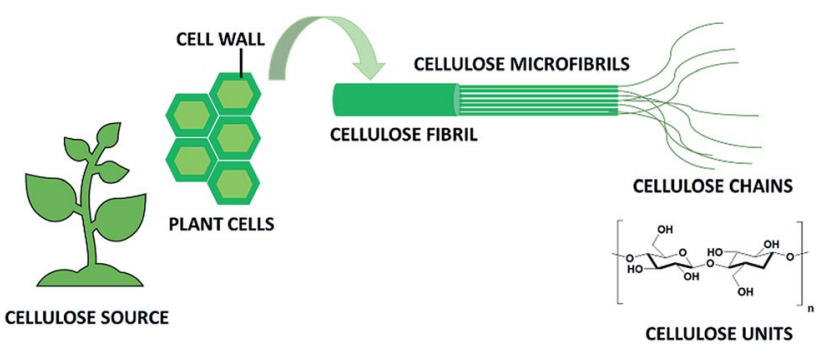

Fig. 1 Schematic representation of cellulose fibrils and microfibrils.
${ }^{a}$ Molecular Design and Synthesis, Department of Chemistry, KU Leuven - University of Leuven, Celestijnenlaan 200F, Box 2404, B-3001 Leuven, Belgium. E-mail: wim. deborggraeve@kuleuven.be

${ }^{b}$ Procter and Gamble Brussels Innovation Center, Temselaan 100, 1853 StrombeekBever, Belgium 
with the term nanocelluloses, which describes cellulosic extracts or processed materials with one dimension in the nanometre range.

Amongst all the methods that can be employed for the mechano-chemical processing of cellulose, ball milling is an emerging technique, which allows avoiding organic solvents. Being easy to use, fast, economical and environmentally friendly, this technique has recently acquired growing interest in chemistry. This paper will focus on the applicability of ball milling for the production and the chemical functionalization on cellulose nanofibers and nanocrystals and will explore recent developments in this field. A variety of research articles and reviews has already been published on the use of ball milling in process engineering, organic synthesis and bio- and polymer nanocomposites. ${ }^{13-17}$ However, the potential of this technique in the field of cellulose nanoparticles has not been fully explored. The aim of this article is to put the existing work into perspective, highlighting the significance and the potential of this green, sustainable technique to facilitate the identification of areas of future development.

\section{The ball mill}

Ball milling is a mechanical technique widely used to grind powders into fine particles and blend materials. ${ }^{18}$ Being an environmentally-friendly, cost-effective technique, it has found wide application in industry all over the world. Since this minireview mainly focuses on the conditions applied for the preparation and functionalisation of nanocellulose derivatives by ball mill, rather than the machinery itself, the different types of machines available in industry will not be herein described. Nevertheless, a general description of the different types of equipment is reported in this section. Depending on the application, there are different types of ball mill. However, it generally consists of a hollow cylindrical shell rotating around its axis, which is partially filled with balls made of e.g. steel, stainless steel, ceramic or rubber (Fig. 2a). It relies on the energy released from impact and attrition between the balls (grinding or milling medium) and the powder. Advantages of this technique include cost-effectiveness, reliability, ease of operation, reproducible results due to energy and speed control, applicability in wet and dry conditions on a wide range of materials (e.g. cellulose, chemicals, fibres, polymers, hydroxyapatite, metal oxides, pigments, catalysts). In contrast, potential disadvantages include: the possibility of contamination, formation of nanomaterials with irregular shape, noise, long milling and cleaning times. Different types of equipment are also available depending on the material to be treated, which have been described in detail in a recent review by Gorrasi and Sorrentino. ${ }^{19}$ In particular they classify the ball mills in two groups depending on their operation mode: direct and indirect milling. In the first case rollers or mechanical shafts directly act on the particles and transfer the kinetic energy. In the second case the kinetic energy is firstly transferred to the mill body and then to the grinding medium. These ball mills are the most widely used in the field of cellulose and they can be further divided into three groups: tumbler ball mills, vibratory mills and planetary mills (Fig. 2b). A tumbler mill consists of a cylinder partially filled with steel balls rotating about its longitudinal axis. In this type of instrument, the efficiency of the process mainly depends on the diameter of the mill. Larger diameters allow greater height of the fall and consequently a higher energy transmitted to the balls. In vibratory mills the vessel containing the sample and the grinding medium are shaken back and forth at high vibrational frequencies. Important factors, in this case, are the vibrational frequency, the amplitude of vibration and the mass of the milling medium. Finally, in a planetary mill the vessels are placed on a rotating supporting disk and they rotate around their own axes. Again, the size of the vessels is an essential parameter for the efficiency of the process as a higher distance allows a higher kinetic energy and therefore stronger impacts.

\section{Effects of milling on the properties of cellulose}

Ball milling has a high influence on the microscopic and macroscopic properties of the resulting material after treatment, such as structure, morphology, crystallinity and thermal stability. The effect of ball mill on the morphological and structural features of cellulose has been described by Okajima and co-workers. ${ }^{20}$ They treated microcrystalline cellulose derived from cotton linters in a planetary ball mill at $200 \mathrm{rpm}$
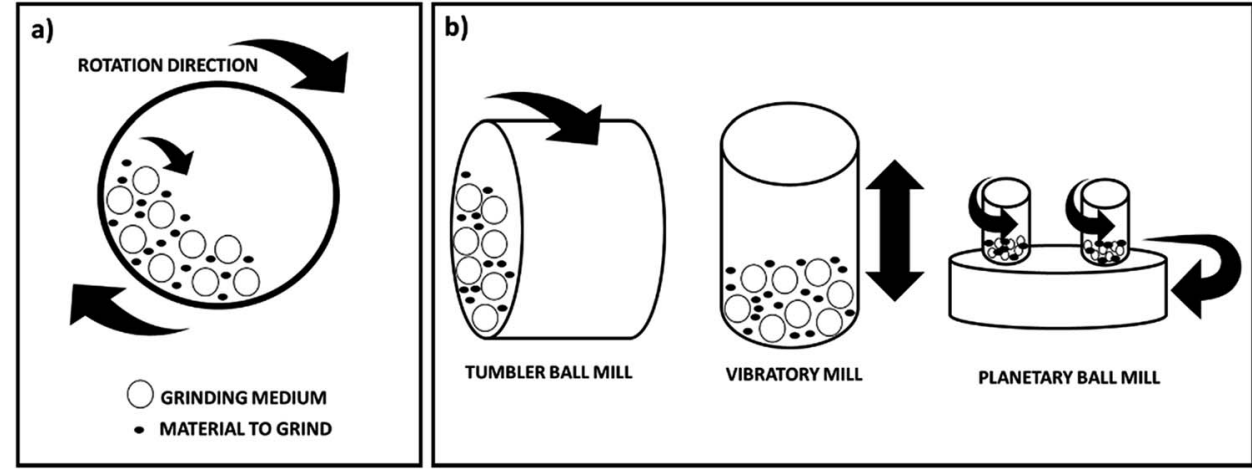

Fig. 2 (a) Schematic representation of a ball mill (horizontal section); (b) different types of instruments (this figure has been adapted from ref. 19 with permission from Royal Society of Chemistry). 
for 4-8 hours in dry and wet conditions with three solvents (water, toluene, 1-butanol). They observed that the different media had an effect on cellulose destruction during the process, leading to variations in morphology, polymorphism and crystallinity. These differences were attributed to the effect of the solvent on the hydrogen bonding amongst the cellulose particles. In dry conditions, aggregated globular particles $(5-10 \mu \mathrm{m}$ diameter) were formed, with a low degree of crystallinity. Globular particles were also obtained in aqueous medium, but the X-ray diffraction profile showed that the crystalline structure was partly converted to cellulose II and retained some cellulose I crystal. When toluene was used as a solvent, plate-like particles were achieved $(<5 \mu \mathrm{m}$ diameter), which maintained the same crystallinity as cellulose I and did not form cellulose II. In the presence of 1-butanol, a mixture of globular and plate-like particles was obtained, which kept their original crystalline structure with some amorphous regions.

A change in morphology of microcrystalline cellulose was also described by Zheng et al., who observed a morphological transformation of microcrystalline cellulose from rod-like to spherical after ball milling was applied. ${ }^{21}$ These particles were employed in combination with starch to produce some pastes, whose rheological properties were remarkably influenced by the concentration of microcrystalline cellulose and the milling time applied to obtain it.

Further research on the effects of milling conditions (i.e. ball-to-cellulose mass ratio, milling time, ball size and alkaline pre-treatment) on the morphology of the prepared nanocellulose derivatives was undertaken by the group of Wang. ${ }^{22,23}$ More in detail, they found out that the size of the milling balls has an impact on the formation of fibrous morphologies rather than particulates. Fibre morphology was also considerably influenced by milling time and ball-to cellulose mass ratio.

Interesting studies on the effects of ball milling on the crystallinity of cellulose were reported by Ago et al., who demonstrated that the mechano-chemical treatment of microcrystalline cotton-derived cellulose in aqueous environment led to the crystalline transformation of cellulose I into the cellulose II polymorph. ${ }^{24}$ Ball milling was performed, in this case, in the presence of $30-50 \%$ water in a planetary equipment at $400 \mathrm{rpm}$ for 2 hours. Further studies on the effect of ball milling on the crystallinity and the surface state of cellulose were undertaken by the Saito group, which observed variations of these parameters in aqueous conditions and in the presence of sodium and potassium chloride salts. ${ }^{25}$ These studies were conducted on microcrystalline cellulose in a tumbling mill machine at $215 \mathrm{rpm}$ for 2-20 hours. The obtained results showed that wet conditions were more effective than dry conditions on the reduction of the degree of crystallinity and the deformation of the sample morphology. Interestingly, the greatest decrease in crystallinity was observed in the sample containing $\mathrm{KCl}$, whereas the crystallinity of the sample containing $\mathrm{NaCl}$ was higher than when the starting material was milled in the absence of salts. This was explained with the fact that $\mathrm{K}^{+}$and $\mathrm{Na}^{+}$ions acted as water-structure breaker and former agents respectively.
The effect of ball milling on the crystallinity index of cellulose was also studied by Ahmad and co-workers. ${ }^{26}$ They treated crystalline cellulose in a planetary ball mill at $600 \mathrm{rpm}$ for 2 hours and studied the differences between the starting material and the final product by a range of techniques including FT-IR, $\mathrm{X}$-ray diffraction, SEM and TGA. The obtained results demonstrated that the ball mill treatment influenced the morphology of the cellulose (which changed to a quasi-circular morphology) and induced a remarkable decrease of crystallinity and thermal stability. Similar conclusions regarding the thermal degradation profile of cellulose were drawn by Hideno, who compared the thermal degradation properties and the crystallinity of commercially available microcrystalline and amorphous cellulose after milling in a planetary instrument for 5-60 minutes. ${ }^{27}$ In particular, in this case, the thermal degradation temperature of crystalline cellulose decreased proportionally with longer milling times. In contrast, this property barely changed in the case of amorphous cellulose.

\section{Preparation and chemical modification of cellulose nanoparticles}

One of the most interesting applications of the ball mill in the field of cellulose is the preparation of CNCs and CNFs (Fig. 3). Being cheap, easy to use and rapid, this method offers several advantages to obtain nanocelluloses and cellulose nanocomposites. ${ }^{28-30}$ However, the applied conditions (e.g. presence of solvents and catalysts, milling time and speed) need to be carefully monitored to avoid excessive grinding of the raw materials. This technique under appropriate conditions and in combination with chemical or enzymatic treatment, can also be employed to induce cellulose hydrolysis and depolymerisation. Since the current article mainly focuses on the extraction and functionalization of nanocelluloses, these applications will not be discussed, but further information can be found in literature. ${ }^{31-37}$

\section{Preparation of cellulose nanocrystals (CNCs)}

Cellulose nanocrystals are usually obtained by hydrolysis of native cellulose with mineral acids, which allows the destruction of the amorphous regions of cellulose and the liberation of the crystalline regions (Fig. 3). This process is facilitated by shearing actions. The ball mill allows applying mechanical

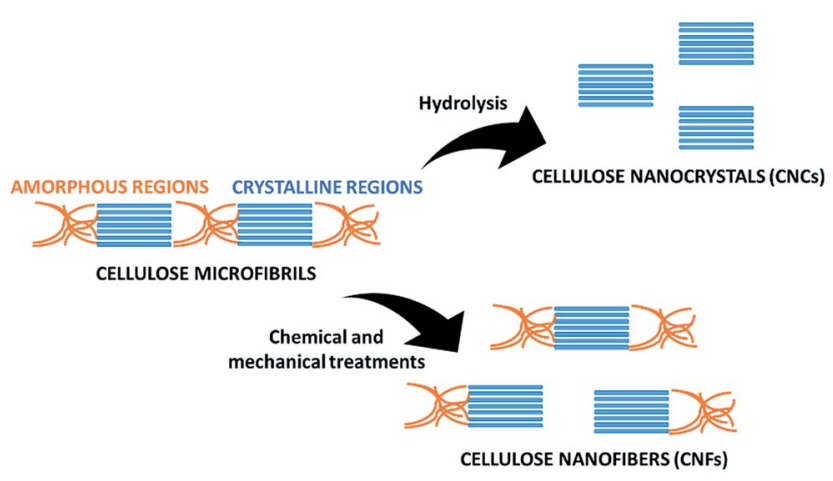

Fig. 3 Production of CNCs and CNFs from cellulose. 
forces in the presence of chemical agents, therefore it represents an ideal method to combine chemical and mechanical actions to obtain CNCs. Factors that should be taken into consideration are the acid selected for hydrolysis, applied pretreatments, time and speed of milling, as they can affect the crystallinity, the morphology and the size of the extracted CNCs.

Lu et al. employed the ball mill to obtain nanocrystals from bamboo pulp by a mechano-chemical treatment in the presence of phosphoric acid. ${ }^{38}$ The pulp was firstly pre-treated using a Fibre Dissociation Device. The obtained material was then ball milled for 1.5-3.5 hours at $400 \mathrm{rpm}$ in the presence of phosphoric acid to give a transparent solution. In this mechanochemical approach, phosphoric acid worked as a swelling agent during cellulose dissolution and facilitated the disintegration of cellulosic fibres into nanofibers. Double distilled water was then added to separate cellulose from a gelatinous precipitate that was also formed. Cellulose nanocrystals were extracted by multiple centrifugations and a subsequent ultrasonication treatment under stirring for $1-3$ hours at $50{ }^{\circ} \mathrm{C}$. The obtained particles were mainly composed of the cellulose type-II polymorph and had a length of 100-200 $\mathrm{nm}$ and a width of 13$30 \mathrm{~nm}$. Subsequently, the same research group designed another method to extract cellulose nanocrystals from bamboo pulp by mechano-chemical activation in the presence of phosphotungstic acid. ${ }^{39}$ Milling was performed for 1.5-2.5 hours, followed by purification and ultrasonication. The resulting nanocrystals had a regular rod-like structure with a curled flat shape, 200-300 nm length and 25-50 nm width. Decisive parameters in the process were the phosphotungstic acid concentration, the reaction time and the milling duration. In particular, at a constant acid concentration, the yield of the isolated CNCs increased with longer reaction times, with optimal conditions after 4.5-5 hours. The yield also increased by increasing the concentration of phosphotungstic acid from 10 to $15 \%$. This facilitated the hydrolysis of the amorphous regions of cellulose and the extraction of the nanocrystals. However, at higher concentrations of acid, the yield of CNCs decreased probably due to synergistic effects of the acid and the ball milling process on the hydrolysis of the crystalline regions of cellulose.

The use of ball milling to obtain cellulose nanocrystals was also explored by the group of $\mathrm{Yu}$, who extracted CNCs from ballmilled wood via a multi-step process. ${ }^{40}$ Douglas-Fir wood chips were firstly hammer-milled to wood flours, which were then treated using a planetary ball mill at $270 \mathrm{rpm}$. This process was followed by enzymatic hydrolysis and treatment with sodium sulphites. The resulting material was then subjected to a delignification process, bleaching and acid hydrolysis in the presence of sulphuric acid. The obtained CNCs had an average length size of $135 \mathrm{~nm}$ and an average width of $6 \mathrm{~nm}$. Compared to the previously described methods, this procedure is more complex and requires several steps, however it also allows the extraction of sugars, lignosulfonates and cellulose through the various steps.

A different method to obtain CNCs was described by Sun and co-workers, who compared the effects of different pretreatments on the morphology and crystallinity of the extracted nanoparticles. ${ }^{\mathbf{4 1}}$ Cellulose from cotton linters was firstly pre-treated by ball milling, freeze drying or mercerization and then hydrolysed or oxidised in the presence of sulphuric acid or TEMPO reagent respectively. Ball milling was performed for 24 hours at $1000 \mathrm{rpm}$. With this pre-treatment, the resulting nanocrystals had a spherical shape with a 100-200 nm diameter and a 3-7 $\mathrm{nm}$ height, rather than a rod-like shape. This demonstrated that the applied pre-treatment was a determining factor in the morphology and particle size of the resulting CNCs. Spherical particles were isolated when mercerization or ball milling were performed, which allowed a higher accessibility compared to the other treatments.

Sphere-shaped nanocrystals were also manufactured by the group of Endo, which described the extraction of CNCs by only applying mechanical actions (i.e. high-pressure homogenization ( $\mathrm{HPH}$ ) and ball milling) without a subsequent chemical treatment from wood-pulp derived cellulose powder. ${ }^{42}$ When the HPH was employed as the sole treatment, rod-like nanowhiskers were isolated with a 200-500 $\mathrm{nm}$ length and a 11$16 \mathrm{~nm}$ width. These were prepared from a $1 \%(\mathrm{w} / \mathrm{v})$ aqueous slurry, which was stirred at room temperature for few days. $\mathrm{HPH}$ was then applied with a total of 10 passes. Spherical particles were extracted when the HPH was preceded by ball milling. In this case, the vacuum-dried powder was ball milled using a planetary instrument at $400 \mathrm{rpm}$ for up to 8 hours with 10 minutes pauses every 10 minutes. The resulting material was then passed through the HPH and subsequently washed with distilled water. The milling time had a remarkable influence on the diameter of the resulting spherical CNCs (40-200 nm) and on the isolated polymorph, since conversion of cellulose I to cellulose II was observed after four hours. This demonstrated that by selecting an appropriate milling time, CNCs of different polymorphs with similar morphology could be obtained.

More recently, Song et al. described a new method to extract CNCs from raw cellulose by ball mill assisted acid hydrolysis. ${ }^{\mathbf{4 3}}$ The starting material was first ground in water using a ball mill at $1300 \mathrm{rpm}$, then treated with oxalic acid. Due to the relatively weak acidity of oxalic acid, ball milling was in this case employed as a pre-treatment to destroy the fibre structure and facilitate the acid hydrolysis. When the reaction was completed, the mixture was centrifuged to isolate the obtained nanocrystals. The yield of the isolated CNCs and their morphology was influenced by several variables including milling time, acid concentration, reaction temperature and time. Interestingly, the authors also considered the economic and commercial aspects of the process and showed that the described method was more cost-effective compared to more traditional ones based on sulphuric acid hydrolysis.

The formation of cellulose crystals by ball mill assisted hydrolysis was also reported by Tian and co-workers, who described the preparation of microcrystalline cellulose (MCC) from eucalyptus pulp by mercerization and subsequent ball mill treatment. ${ }^{44}$ The pulp was first treated with sodium hydroxide, then milled in the presence of the same at $450 \mathrm{rpm}$ for 5 hours. The obtained MCC was combined with starch to obtain reinforced film composites with improved mechanical strength compared to starch. 


\section{Functionalisation of CNCs}

As previously mentioned, one of the advantages of ball milling is that it allows applying mechanical shearing actions in the presence of chemical agents. For this reason, this technology can be exploited not only for the extraction of cellulose nanocrystals, but also to functionalise them. Optimisation of the applied method, however, is of fundamental importance since the yield and the degree of substitution (DS) of the isolated crystals is affected by milling duration and rotation speed.

Tang et al., for example, described a solvent-free method to extract and chemically modify CNCs in the presence of maleic anhydride. ${ }^{45}$ This process was applied on cellulose pulp from filter paper, which was mechano-chemically treated in a planetary ball mill with maleic anhydride and sulphuric acid at $500 \mathrm{rpm}$ for $0.5-2$ hours. This was followed by treatment in an ultrasonic reactor to improve the efficiency of the chemical reaction. The treatment with the ball mill had an effect on the yield (i.e. the percentage of nanocrystals extracted from cellulose pulp) and the degree of substitution of the resulting CNCs. When ball milling was not applied, only a $16.3 \%$ yield was achieved, whereas, after milling for $0.5-1$ hour, the yield improved to 56.1-61.1\% probably due to breakage of inter- and intra- molecular hydrogen bonds, with a consequent increase of hydroxyl groups available to react. It was noted that rising the reaction time to 2 hours resulted in an excessive destruction of the cellulose crystalline structure and in a decrease of the yield to $54.7 \%$. A similar effect was observed for the DS values, which improved by increasing the milling time to one hour and subsequently decreased when the process duration was extended to two hours. The esterification of the most accessible regions took place first, followed by functionalization of the less reachable parts of the crystals. Variations in crystallinity were also observed. In particular, an increase in crystallinity was detected due to the degradation of the amorphous regions of cellulose during the performed reactions.

The manufacturing and functionalization of cellulose nanocrystals by ball milling was also described by Lu et al., who reported the mechano-chemical treatment and esterification of bamboo fibres in the presence of 4-dimethylaminopyridine (DMAP) as a catalyst. ${ }^{46}$ The cellulose bamboo pulp was ball milled with DMAP at $500 \mathrm{rpm}$ for 1-3 hours. After the reaction, the mixture was sonicated and purified by multiple centrifugations with double distilled water at $10.000 \mathrm{rpm}$ for 15 minutes. This method allowed obtaining short rod-like CNCs with an average length of 130-230 $\mathrm{nm}$ and an average width of 20-40 nm. Interestingly, the strong hydrogen bonding among the nanocrystals led to the formation of web-like self-assembled networks. In this case, the mechanical treatment did not cause a change in the cellulose polymorphism. However, variations in the crystallinity were observed, which increased from $63.5 \%$ to $71.3 \%$ by increasing the milling time from 1 to 2 hours and decreased to $67.3 \%$ after 3 hours, probably due to excessive destruction of the crystalline regions. The functionalized CNCs kept a high crystallinity index, confirming that the chemical modification mainly occurred at the surface or in the amorphous regions of cellulose. Furthermore, variations in the yield and in the DS of the obtained
CNCs were observed. In particular, an increase in the yield from $17.45 \%$ to $43.06 \%$ and in the DS values was observed when the duration of milling was brought from 1 to 2 hours. This was explained with the action of the ball mill on the inter- and intramolecular hydrogen bonds, which were cleaved leading to a higher number of hydroxyl groups available to react. When 3 hours milling time was applied, the yield decreased to $34.37 \%$ together with the DS, probably due to excessive destruction of the crystalline regions.

\section{Preparation of cellulose nanofibers (CNFs)}

Cellulose nanofibers are usually prepared by a combination of chemical and mechanical treatments, which allow extracting the nanofibers after removal of lignin and hemicellulose (Fig. 3). ${ }^{47}$ The use of the ball milling technology to obtain CNFs has been only partially explored. Most of the reported studies were performed in wet conditions, as they were found to be optimal for fibrillation. This is mainly due to the fact that fibre swelling in water or organic solvents allows the disruption of hydrogen bonds avoiding excessive grinding of the starting materials. Factors to be taken into consideration are milling time, rotation speed and solvent employed, as they have an influence on fibrillation, crystallinity, size, morphology and thermal degradation properties of the isolated fibres.

The group of Okamoto described the production of cellulose nanofibers from commercially available cellulose powder in aqueous medium. ${ }^{48}$ The starting material was firstly pulverized and then fibrillated by milling at three different rotational rates (150, 200 and $250 \mathrm{rpm})$. The pulverization time affected the particle size, which diminished with longer milling times. When pulverization was performed in the presence of a higher water content, fibrillation improved due to disruption of hydrogen bonds and fibre swelling. The resulting fibres were employed as reinforcement in wood flour board to replace chemical adhesives.

Ball milling in aqueous conditions was also employed by the Wang group to obtain cellulose nanofibers from wood pulp. ${ }^{49} \mathrm{In}$ particular, they first treated dried the soft wood pulp in water at room temperature to loosen the hydrogen bonding between the fibres, then they milled the resulting loosened fibres in aqueous medium for 2 hours. The isolated NFCs had an average diameter of $32 \mathrm{~nm}$ and a rather flat shape, as shown by SEM. Later on, the same research group employed this preparation method to obtain NFCs, which were freeze-dried and used for the fabrication of carbon nanofibers by pyrolysis. ${ }^{50}$ The NFCs had a diameter below $100 \mathrm{~nm}$ and the morphology of the resulting carbon nanofibers was influenced by the applied drying method. Further studies conducted on ball milled NFCs in aqueous media showed that the different dimensions of the nanofibers had a remarkable influence on the fibre ability to form sheets by different methods. ${ }^{51}$ The samples with a more heterogeneous distribution of fibres displayed a better ability to form sheets compared to more homogeneous samples.

In contrast, Nuruddin et al. applied ball milling after chemical treatments. ${ }^{52}$ The cellulose used as a starting material in this case was extracted from wheat straw and kenaf fibers via 
formic acid/peroxyacetic acid and hydrogen peroxide treatment. Milling was then performed in 80\% ethanol for 30-120 minutes using a high-energy ball mill. The mechanical treatment resulted in a reduction of the fibre length and diameter, probably due to degradation of the cellulose amorphous regions. Fibrillation was helped by the wet environment, which facilitated the intra-fibre swelling. Longer milling times enhanced the breaking of the hydrogen bonds, inducing a higher separation of the resulting nanofibers, and a $13-17 \%$ decrease in crystallinity. The diameter of the resulting CNFs was in the range of 21-30 $\mathrm{nm}$. In this case, the thermal stability of the cellulose nanofibers did not change significantly after ball milling.

Militky and co-workers described the preparation of CNFs from jute fibres in aqueous conditions. ${ }^{53}$ Jute was firstly chemically treated to remove the lignin and hemicellulose. The resulting material was then initially pulverized using a planetary mill in a dry environment (10 minutes) and subsequently in wet conditions ( 3 hours) at $850 \mathrm{rpm}$. Interestingly, the absence of a dispersion medium had an impact on the size of the resulting CNFs. In particular, ball milling in dry conditions led to a wider size distribution since the material was sticking to the surface of the container and formed aggregates due to the presence of surface interactions in the dry state. Particle size in this case was around $500 \mathrm{~nm}$.

The preparation of CNFs from jute nanofibers was also described by the group of Mishra, which ball milled chemically pre-treated jute fibres with a planetary instrument first at $850 \mathrm{rpm}$ in dry conditions (3 minutes) and then aqueous conditions (one hour). ${ }^{54}$ The fibre size, in this case, was below $500 \mathrm{~nm}$. They were employed for the recovery of mercury ions from aqueous solution. Smaller jute nanofibers were obtained by Padal et al., which used a planetary ball mill at 100-200 rpm for a longer period of time (10-80 hours) in an aqueous environment. ${ }^{55}$ The width of the resulting fibres was within the range of 20-52 $\mathrm{nm}$. The experiments were carried out at different rotational speeds, milling times and using different ball sizes. Optimal conditions to achieve better defibrillation were observed at the highest milling speed (200 rpm) with $3 \mathrm{~mm}$ diameter balls after 20 hours.

A different method to extract cellulose nanofibers was reported by Phanthong et al., who applied ball milling as a pretreatment, followed by acid hydrolysis. ${ }^{56}$ Commercially available cellulose paper was treated in a planetary instrument at $300 \mathrm{rpm}$ for $0.5-3$ hours. Acid hydrolysis was then performed using sulphuric acid (47 wt\%) for 90 minutes at $45{ }^{\circ} \mathrm{C}$. The ball mill worked by cracking the cellulose microfibrils along their longitudinal axis leading to nanofibers with a shorter diameter and length when longer milling times were applied. The mechanical pre-treatment influenced the crystallinity and crystal sizes of the CNFs as the amorphous regions were degraded and removed. The thermal degradation profile of the fibres was also affected, as the collected CNFs had a lower decomposition temperature compared to those that were not ball milled.

More recently the same research group described a method to extract cellulose nanofibers in high yield $(93.1 \%)$ in the presence of the ionic liquids 1-butyl-3-methylimidazolium chloride (BMIMCl) and 1-ethyl-3-methylimidazolium acetate
(EMIMOAc) ${ }^{57}$ These ionic liquids were chosen since they can easily dissolve cellulose. Ball milling was performed on cellulose pulp using a planetary instrument at $400 \mathrm{rpm}$ for two hours. Purification was then undertaken by washing with distilled water. After the mechanical treatment was applied, the resulting fibrils maintained the same crystal structure as the starting material (cellulose I), while crystallinity and crystal size decreased depending on the employed ionic liquid. In particular, the CNFs extracted using EMIMOAc had a lower crystallinity, crystal size, thermal stability and yield. This was attributed to the higher ability of this medium to interact with the hydroxyl groups of cellulose compared to BMIMCl. Despite the cost of the ionic liquids, which are notoriously expensive, this method allowed the recovery of the medium, which could be re-used at least four times.

Further studies conducted on wet milling demonstrated that the type of media employed for milling had an impact on the type of nanoparticles obtained. This was reported by Zhao and co-workers, who studied the effect of milling in dry conditions, aqueous medium and silicone oil (PDMS). ${ }^{58}$ They used commercially available cellulose powder from cotton linters as a starting material. This was milled using a planetary instrument in dry conditions or in a hydrophilic or hydrophobic environment. The process was conducted at a rotational speed of $400 \mathrm{rpm}$ for 12 hours. Aggregated globular particles were obtained by dry milling due to the fast decrystallisation, whereas nanofiber dispersions were achieved in wet conditions. Interestingly, milling in the presence of PDMS led to the formation of nanosheets with a specific orientation. This was explained with the alignment of the elementary fibrils induced by the mechanical impacts in a hydrophobic environment.

More recently, Harini et al. described the production of CNFs from banana peel by ball milling in DMSO as a dispersion medium. ${ }^{59}$ The raw material was first exposed to a micro-wave assisted pre-treatment that allowed the isolation of micro cellulose fibres. This was followed by milling in a high-energy ball milling machine for 2 hours in DMSO and subsequent ultrasonication for one hour to disintegrate the amorphous regions within the fibres. The resulting nanofibers had a diameter within $100 \mathrm{~nm}$ and were characterised by a range of techniques including dynamic light scattering, thermal gravimetric analysis, differential scanning calorimetry, X-ray diffraction and microscopy. These were then chemically modified by esterification to give the corresponding cellulose acetate and laurate derivatives.

The use of ball milling as a defibrillation method after a series of pre-treatments of raw materials was also described by the group of Elkoun, who reported the extraction of CNFs and CNCs from carrot pulp by ball milling and acid hydrolysis respectively. ${ }^{60}$ More in detail, NFCs were obtained from never dried carrot pulp (4\%) by ball milling with zirconium balls at $2400 \mathrm{rpm}$ at room temperature for different grinding times ranging from 1.5 to 5 minutes. The resulting nanofibrils had a width of $2-18 \mathrm{~nm}$, as shown by TEM and AFM. Interestingly, in contrast with other described processes, in this case, ball milling did not affect the crystallinity of the carrot fibres. However, they displayed a lower thermal stability compared to raw fibres and bleached carrot fibres. 


\section{Functionalisation of CNFs}

As previously described for CNCs, since the ball mill allows applying mechanical shearing actions in the presence of chemical agents, it can also be used to prepare chemically modified cellulose nanofibrils. The surface properties of fibres, their size, morphology, thermal stability and the percentage of functionalization are influenced by milling time and speed, solvent, reagent quantities and applied pre-treatments. Although a number of studies demonstrated the applicability of the ball milling technology for this purpose, it has not been fully exploited and, so far, to the best of our knowledge, esterification is the only reported type of functionalization for CNFs using ball milling.

Huang et al. described the simultaneous application of mechanical and chemical actions to obtain surface esterified cellulose nanofibers. ${ }^{61}$ This process was conducted on microcrystalline cellulose for 16 hours in a planetary ball mill using hexanoyl chloride as an esterifying agent. This was followed by centrifuge washing, sonication in DMF and solvent exchange in THF. The success of the esterification reaction was confirmed by FTIR, which showed a gradual increase in the intensity of the stretching band of the ester bond with time. After ball milling, the DS was estimated to be 0.58 . The integrity of the nanofibrillar structures was confirmed by microscopy techniques, which showed an interconnected network of fibrils with an average width of 15-20 nm. Further studies conducted by the same research group showed that an aqueous pre-treatment was beneficial for the dispersion of the starting material prior to the chemical-mechanical process and to facilitate the esterification reaction. ${ }^{62}$ The pre-treatment was performed by immerging the starting material in water or in a $2 \%$ solution of $\mathrm{NaOH}$ for three days, followed by a solvent exchange in DMF. The esterification reaction was then performed in a planetary ball mill at $200 \mathrm{rpm}$ using hexanoyl chloride as an esterifying agent. This process was performed including a 10 minutes cooling time every 20 minutes of milling. Compared to the previously reported method, the water pre-treatment allowed reducing the milling time from 16 to 8 hours. Different starting materials were used in this study: microcrystalline cellulose, ashless pulp and kraft pulp. Differences in the resulting fibre lengths and widths were attributed to the different starting materials.

Subsequently, they also reported the preparation and functionalization of NFCs with $n$-dodecyl succinic anhydride and succinic anhydride. ${ }^{63}$ Microcrystalline cellulose and hardwood pulp were used as starting materials. Ball milling was performed in the presence of DMAP as a catalyst at $200 \mathrm{rpm}$ for 2-40 hours with an interval of two minutes every 20 minutes of working time. The resulting nanofibrils had a length of several $\mu \mathrm{m}$ and a width in the $15-20 \mathrm{~nm}$ range. The formation of the ester bonds was monitored by FTIR and the DS measured by conductometric titration. In particular, the maximum DS for the functionalization with succinic anhydride was obtained after 30 hours of milling (0.31). In contrast, the reaction with $n$-dodecyl succinic anhydride gave a maximum DS value after 40 hours (0.23). Variations in surface properties of the fibres and in thermal stability were observed and related to the mechanical treatment and to the different ester functionalities inserted.

Manufacturing and chemical modification of cellulose nanofibers with the ball mill was also described by the group of Fu. ${ }^{64}$ Nanofibrillated cellulose was obtained from wood pulp by a number of homogenization processes. It was then dispersed in DMF. This suspension was milled using a planetary instrument at $500 \mathrm{rpm}$ for 3 hours in the presence of hexanoyl chloride and purified by centrifugation. By controlling the milling duration and the reagent quantities, CNFs with different DS values and sizes were obtained. These materials were used to produce films, which displayed enhanced tensile strength and elongation at break in comparison to unmodified cellulose due to disentanglement or to higher aspect ratio of fibres. At higher DS values, the mechanical properties further improved due to improved dissolution of cellulose, which generated a continuous phase surrounding undissolved fibres.

The same reaction in the presence of hexanoyl chloride was also described by Kang et al., who used corncob cellulose as a starting material. ${ }^{65}$ The isolated products were employed to produce cellulose nanopaper with high optical transparency and tensile strength. Interestingly, the IR studies conducted during ball milling demonstrated that the fibrillation of cellulose and its esterification took place simultaneously leading to a rapid growth of the DS during the first 12 hours, which reached a maximum value of 0.96 after 24 hours.

As mentioned before, esterification is, as far as we know, the reaction that has been most described. This type of modification was also reported by Rao et al., who used ball mill-assisted cellulose functionalization to induce surface fluorination. ${ }^{66}$ The reaction was carried out on commercially available cellulose powder in the presence of pentafluorobenzoylchloride (PFBC) as an esterification agent and pyridine as a catalyst in DMF or toluene. Ball milling was performed at $200 \mathrm{rpm}$ for 12 hours, followed by purification by multiple centrifugations in toluene, DCM and ethanol. In this case, the employed organic medium had a strong influence on the percentage of functionalization and on the microscopic morphology of the obtained cellulose fibres. Milling in DMF allowed the formation of highly surfacefunctionalised nanofibers, whereas, when toluene was used as a dispersion medium, compacted platelets with low DS values were obtained. The employed solvent also had a remarkable influence on crystallinity. Milling in DMF allowed maintaining cellulose crystallinity, whereas in toluene, a decrystallisation similar to dry milling was observed. Both solvents induced a high degree of hydrophobization of the resulting material.

\section{Fabrication of CNCs and CNFs nanocomposites via ball milling}

Polymer nanocomposites are defined as a class of two-phase materials of whom one of the two has one dimension in the nanometre range. ${ }^{67,68}$ These materials offer the possibility to improve and tailor specific properties of the mixture components, hence expanding the scope of what can be achieved with them. Due to its broad availability, variety of properties and 
cost-effectiveness, nanocelluloses are interesting candidates as fillers to form nanocomposites in association with other polymers. By allowing the application of mechanical shearing actions in the presence of chemical agents (e.g. cross-linkers), the ball mill represents an easy method to prepare such hybrid systems. ${ }^{69}$ One example has been reported by Wang et al., who prepared a polymer nanocomposite from cellulose nanocrystals and ultrahigh molecular weight polyethylene (UHMWPE). ${ }^{70}$ This nanocomposite was obtained by mixing commercially available CNCs with UHMWPE in a planetary ball mill at $200 \mathrm{rpm}$ rotational speed for six hours. The resulting mixture was then hot-pressed to form disk-like or dumbbell shaped derivatives, which had an improved biocompatibility and could therefore be a promising alternative to UHMWPE as a bearing material for artificial joints.

Further examples were reported by Lagaron and co-workers, who employed the ball mill to obtain nanocomposites of CNCs and poly(3-hydroxybutyrate-co-3-hydroxyvalerate) (PHBV). ${ }^{71}$ The cellulose nanocrystals used as starting materials were obtained from bacteria and ground to a powder. These were milled together with PHBV using a centrifugal ball mill at $650 \mathrm{rpm}$ for 60 minutes. The resulting composites were then moulded in a hot press to form thick films. The dispersion of the CNCs within the polymer matrix was studied by SEM, which showed a good dispersion and distribution of the crystals within the nanocomposites. Compared to PHBV, the nanocomposites exhibited better thermal stability, crystallinity, oxygen permeability and a reduction of water vapour diffusion.

In a subsequent study, they also combined CNCs from bacterial sources with polylactic acid (PLA). ${ }^{72}$ Also in this case, the nanocrystals were firstly ground and then mixed with the polymer using a centrifugal ball mill under the same conditions described in the previous study. Thick films were produced by applying a hot press. The investigations conducted on the resulting films showed that the CNCs were well dispersed within the PLA matrix, with some residual aggregates, which increased in number at higher concentrations of cellulose nanocrystals. The presence of CNCs within the polymeric network resulted in an increase in the crystallinity of the resulting nanocomposite. However, the thermal properties of the materials were not significantly affected by the ball milling treatment. In comparison to PLA, the resulting nanocomposite also displayed better oxygen permeability and thermomechanical properties.

Polymer nanocomposites were also obtained by milling cellulose nanofibrils with other polymers. ${ }^{67}$ An example was reported by Abraham et al., who prepared a natural rubber/CNF composite. ${ }^{73}$ The cellulose fibres used in this study were obtained by steam explosion of banana fibre and mixed with natural rubber in the presence of different cross-linkers and water using a planetary ball mill at $300 \mathrm{rpm}$ for two hours followed by ultra-sonication. The resulting composite was studied by SEM and AFM, which showed that the CNFs were coated with the natural rubber latex and homogeneously distributed within the polymeric matrix with some exceptions due to the formation of aggregates. It was also observed that the milling process caused an increase in the amorphous regions of the cellulose nanofibers. This enhanced the availability of free hydroxyl groups due to the breakage of intra- and inter-molecular hydrogen bonds, thus making the polysaccharide more reactive. The resulting composite also displayed variations in the mechanical properties compared to the lone components. In particular, compared to natural rubber, the addition of cellulose nanoparticles induced a decrease of the elongation break and significant increase of the Young modulus and mechanical strength.

In a subsequent study, they also investigated the barrier properties of such nanocomposites in the presence of organic solvents such as $p$-xylene and toluene. ${ }^{74}$ Again the cellulose nanofibers were found to be homogeneously dispersed within the rubber matrix. Interestingly, it was found that the diffusion coefficient of the loaded samples was higher at low CNFs concentrations, probably due to stronger interactions between the two polymers, which hindered the release of the cargo through the membrane.

Further studies on the same nanocomposite were also carried out by Thomas and co-workers, who prepared the polymer mixture from nanocellulose fibres extracted from jute. ${ }^{75}$ The method applied was the same as described above and included a ball mill treatment for two hours at $300 \mathrm{rpm}$ in aqueous conditions together with different cross-linkers, followed by ultra-sonication. XRD and TEM confirmed that CNFs was homogeneously dispersed within the rubber matrix. The mechanical strength of the obtained nanocomposites was higher at higher concentrations of cellulose fibres, due to a stronger network formed by the CNFs within the other polymer matrix. This was accompanied by an increase in the Young modulus and tensile strength and a decrease of the elongation at break.

In a recent work reported by Deng et al., a poly $(\varepsilon-$ caprolactone)/functionalized cellulose nanofibers (PCL/f-NFC) nanocomposite was produced by mechanical and chemical treatment in a ball mill. ${ }^{76}$ The two polymers were combined to give a composite with enhanced rheological properties. Functionalised NFCs were prepared by reaction with hexanoyl chloride in DMF by ball milling for 3 hours at $500 \mathrm{rpm}$. The increase in hydrophobicity of the cellulose fibres after functionalisation allowed a good dispersion in PCL and the formation of polymer composite films. The final materials displayed a higher tensile strength and elongation at break compared to PCL.

\section{Conclusion}

Ball milling is a simple, fast, cost-effective green technology with enormous potential. The studies reviewed showed its applicability to prepare and chemically functionalise cellulose nanocrystals and nanofibers. One of the main advantages of this method is the possibility to combine it with chemical treatments, thus allowing obtaining the desired products with minimal effort. Nevertheless, a number of factors has to be taken into consideration, which can affect the features of the isolated nanocelluloses (e.g. milling time, speed, type of solvent, pre-treatments). Optimisation of the method is therefore of fundamental importance to isolate CNCs and CNFs with the desired properties. Features that can be affected include 
crystallinity, thermal degradation, length, width and morphology of the isolated nanoparticles. However, it should not be forgotten that the source of the raw starting material can also have a considerable impact on the efficiency of the method and the properties of the extracted nanocelluloses and therefore it should be carefully selected.

Although ball milling technology has been exploited in process engineering, organic synthesis and the formation of nanocomposites, its potential to isolate and chemically modify cellulose nanoparticles has not been fully explored, as only a limited number of studies has been carried out. In particular, further studies on the functionalisation of CNCs and CNFs would be of great interest, as it is often problematic to react these materials, due to their low solubility in water and organic solvents. To our knowledge, esterification is the most commonly reported reaction; however, other types of chemical modification could be explored, although limited by the functional groups present. Options, for example, could be other reactions where the cellulosic hydroxyl groups act as a nucleophile. Moreover, since ball milling can be employed in dry and aqueous conditions, it could represent a green, sustainable technology for industrial application. Further studies on the preparation of cellulose-based nanocomposites would also be of interest as this technique offers a simple, fast way to obtain such hybrid systems.

Since this is a promising technology, we hope that this minireview will help to understand its significance and potential and will facilitate the identification of areas of future development.

\section{Conflicts of interest}

There are no conflicts of interest to declare.

\section{Acknowledgements}

The authors would like to thank the FP7-PEOPLE-2013-IAPPEU Programme, for providing financial support to the research project ISSFLOW-FP7-PEOPLE-2013-IAPP-612330.

\section{References}

1 M. P. Wilson and M. R. Schwarzman, Green Chemistry: Terminology and Principles Response, Environ. Health Perspect., 2009, 117(10), A386-A434.

2 P. T. Anastas and M. M. Kirchhoff, Origins, current status, and future challenges of green chemistry, Acc. Chem. Res., 2002, 35(9), 686-694.

3 R. A. Sheldon, Green and sustainable manufacture of chemicals from biomass: state of the art, Green Chem., 2014, 16(3), 950-963.

4 T. Abitbol, A. Rivkin, Y. F. Cao, Y. Nevo, E. Abraham, T. BenShalom, S. Lapidot and O. Shoseyov, Nanocellulose, a tiny fiber with huge applications, Curr. Opin. Biotechnol., 2016, 39, 76-88.

5 M. A. Hubbe, A. Ferrer, P. Tyagi, Y. Y. Yin, C. Salas, L. Pal and O. J. Rojas, Nanocellulose in Thin Films, Coatings, and Plies for Packaging Applications: A Review, Bioresources, 2017, 12(1), 2143-2233.

6 D. Klemm, F. Kramer, S. Moritz, T. Lindstrom, M. Ankerfors, D. Gray and A. Dorris, Nanocelluloses: A New Family of Nature-Based Materials, Angew. Chem., Int. Ed., 2011, 50(24), 5438-5466.

7 R. J. Moon, A. Martini, J. Nairn, J. Simonsen and J. Youngblood, Cellulose nanomaterials review: structure, properties and nanocomposites, Chem. Soc. Rev., 2011, 40(7), 3941-3994.

8 C. Salas, T. Nypelo, C. Rodriguez-Abreu, C. Carrillo and O. J. Rojas, Nanocellulose properties and applications in colloids and interfaces, Curr. Opin. Colloid Interface Sci., 2014, 19(5), 383-396.

$9 \mathrm{H}$. Xie, H. Du, X. Yang and C. Si, Recent Strategies in Preparation of Cellulose Nanocrystals and Cellulose Nanofibrils Derived from Raw Cellulose Materials, Int. J. Polym. Sci., 2018, 2018, 7923068.

10 A. Naderi, Nanofibrillated cellulose: properties reinvestigated, Cellulose, 2017, 24(5), 1933-1945.

11 A. F. Jozala, L. C. de Lencastre-Novaes, A. M. Lopes, V. D. Santos-Ebinuma, P. G. Mazzola, A. Pessoa, D. Grotto, M. Gerenutti and M. V. Chaud, Bacterial nanocellulose production and application: a 10-year overview, Appl. Microbiol. Biotechnol., 2016, 100(5), 2063-2072.

$12 \mathrm{H}$. Ullah, F. Wahid, H. A. Santos and T. Khan, Advances in biomedical and pharmaceutical applications of functional bacterial cellulose-based nanocomposites, Carbohydr. Polym., 2016, 150, 330-352.

13 A. Stolle, T. Szuppa, S. E. S. Leonhardt and B. Ondruschka, Ball milling in organic synthesis: solutions and challenges, Chem. Soc. Rev., 2011, 40(5), 2317-2329.

14 A. Stolle, W. Hopfe and W. Simon, Planetary Ball Mills as Innovative Reactors in Organic Synthesis Laboratory, Chem.-Ing.-Tech., 2013, 85(6), 914-918.

15 A. Stolle, Technical Implications of Organic Syntheses in Ball Mills, RSC Green Chem. Ser., 2015, 31, 241-276.

16 M. O. M'hamed, Ball Milling for Heterocyclic Compounds Synthesis in Green Chemistry: A Review, Synth. Commun., 2015, 45(22), 2511-2528.

17 L. Takacs, Self-sustaining reactions induced by ball milling, Prog. Mater. Sci., 2002, 47(4), 355-414.

18 F. Moosakazemi, M. R. T. Mohammadi, M. Mohseni, M. Karamoozian and M. Zakeri, Effect of design and operational parameters on particle morphology in ball mills, Int. J. Miner. Process., 2017, 165, 41-49.

19 G. Gorrasi and A. Sorrentino, Mechanical milling as a technology to produce structural and functional bionanocomposites, Green Chem., 2015, 17(5), 2610-2625.

$20 \mathrm{M}$. Ago, T. Endo and K. Okajima, Effect of solvent on morphological and structural change of cellulose under ball-milling, Polym. J., 2007, 39(5), 435-441.

$21 \mathrm{Y}$. Zheng, Z. Fu, D. Li and M. Wu, Effects of Ball Milling Processes on the Microstructure and Rheological Properties of Microcrystalline Cellulose as a Sustainable Polymer Additive, Materials, 2018, 11(7), 1057. 
22 L. Zhang, T. Tsuzuki and X. Wang, Preparation of cellulose nanofiber from softwood pulp by ball milling, Cellulose, 2015, 22(3), 1729-1741.

23 L. Zhang, Y. Jia, H. He, J. Yin, R. Chen, C. Zhang, W. Shen and $\mathrm{X}$. Wang, Multiple factor analysis on preparation of cellulose nanofiber by ball milling from softwood pulp, BioResources, 2018, 13(2), 2397-2410.

24 M. Ago, T. Endo and T. Hirotsu, Crystalline transformation of native cellulose from cellulose I to cellulose II polymorph by a ball-milling method with a specific amount of water, Cellulose, 2004, 11(2), 163-167.

25 S. Nemoto, T. Ueno, A. Watthanaphanit, J. Hieda and N. Saito, Crystallinity and surface state of cellulose in wet ball-milling process, J. Appl. Polym. Sci., 2017, 134(22), 44903.

26 A. S. Khan, Z. Man, M. A. Bustam, C. F. Kait, M. I. Khan, N. Muhammad, A. Nasrullah, Z. Ullah and P. Ahmad, Impact of Ball-Milling Pretreatment on Pyrolysis Behavior and Kinetics of Crystalline Cellulose, Waste Biomass Valorization, 2016, 7(3), 571-581.

27 A. Hideno, Comparison of the Thermal Degradation Properties of Crystalline and Amorphous Cellulose, as well as Treated Lignocellulosic Biomass, Bioresources, 2016, 11(3), 6309-6319.

28 P. Phanthong, P. Reubroycharoen, X. Hao, G. Xu, A. Abudula and G. Guan, Nanocellulose: Extraction and application, Carbon Resources Conversion, 2018, 1(1), 32-43.

29 T. Hirotsu, T. Endo and M. Umemura, Ball-milling promoted chemical bonding between cellulose and plastics, in Green Chemistry, RSC, 2015, vol. 2015, pp. 203-240.

30 M. Rajinipriya, M. Nagalakshmaiah, M. Robert and S. Elkoun, Importance of Agricultural and Industrial Waste in the Field of Nanocellulose and Recent Industrial Developments of Wood Based Nanocellulose: A Review, ACS Sustainable Chem. Eng., 2018, 6(3), 2807-2828.

31 Y. J. Zhang, Q. Li, J. M. Su, Y. Lin, Z. Q. Huang, Y. H. Lu, G. S. Sun, M. Yang, A. M. Huang, H. Y. Hu and Y. Q. Zhu, A green and efficient technology for the degradation of cellulosic materials: Structure changes and enhanced enzymatic hydrolysis of natural cellulose pretreated by synergistic interaction of mechanical activation and metal salt, Bioresour. Technol., 2015, 177, 176-181.

32 L. S. Ribeiro, J. J. M. Orfao and M. F. R. Pereira, Enhanced direct production of sorbitol by cellulose ball-milling, Green Chem., 2015, 17(5), 2973-2980.

33 U. Mais, A. R. Esteghlalian, J. N. Saddler and S. D. Mansfield, Enhancing the enzymatic hydrolysis of cellulosic materials using simultaneous ball milling, Biotechnol. Appl. Biochem., 2002, 98, 815-832.

34 R. Schmidt, S. Fuhrmann, L. Wondraczek and A. Stolle, Influence of reaction parameters on the depolymerization of $\mathrm{H}_{2} \mathrm{SO}_{4}$-impregnated cellulose in planetary ball mills, Powder Technol., 2016, 288, 123-131.

35 Q. H. Zhang and F. Jerome, Mechanocatalytic Deconstruction of Cellulose: An Emerging Entry into Biorefinery, Chemsuschem, 2013, 6(11), 2042-2044.
36 J. Hilgert, N. Meine, R. Rinaldi and F. Schuth, Mechanocatalytic depolymerization of cellulose combined with hydrogenolysis as a highly efficient pathway to sugar alcohols, Energy Environ. Sci., 2013, 6(1), 92-96.

37 A. Onda, T. Ochi and K. Yanagisawa, Selective hydrolysis of cellulose into glucose over solid acid catalysts, Green Chem., 2008, 10(10), 1033-1037.

38 Q. L. Lu, W. Y. Lin, L. R. Tang, S. Q. Wang, X. R. Chen and B. Huang, A mechanochemical approach to manufacturing bamboo cellulose nanocrystals, J. Mater. Sci., 2015, 50(2), 611-619.

39 Q. L. Lu, Z. H. Cai, F. C. Lin, L. R. Tang, S. Q. Wang and B. A. Huang, Extraction of Cellulose Nanocrystals with a High Yield of $88 \%$ by Simultaneous Mechanochemical Activation and Phosphotungstic Acid Hydrolysis, Energy Environ. Sci., 2016, 4(4), 2165-2172.

40 L. X. Du, J. W. Wang, Y. Zhang, C. S. Qi, M. P. Wolcott and Z. M. Yu, A co-production of sugars, lignosulfonates, cellulose, and cellulose nanocrystals from ball-milled woods, Bioresour. Technol., 2017, 238, 254-262.

41 D. Yang, X. W. Peng, L. X. Zhong, X. F. Cao, W. Chen and R. C. Sun, Effects of pretreatments on crystalline properties and morphology of cellulose nanocrystals, Cellulose, 2013, 20(5), 2427-2437.

42 T. T. Nge, S. H. Lee and T. Endo, Preparation of nanoscale cellulose materials with different morphologies by mechanical treatments and their characterization, Cellulose, 2013, 20(4), 1841-1852.

43 K. Song, Y. Ji, L. Wang, Y. Wei and Z. Yu, A green and environmental benign method to extract cellulose nanocrystal by ball mill assisted solid acid hydrolysis, J. Cleaner Prod., 2018, 196, 1169-1175.

44 Y. Tian, K. Zhang, M. Zhou, Y. Wei, F. Cheng, Y. Lin and P. Zhu, High-Performance Starch Films Reinforced With Microcrystalline Cellulose Made From Eucalyptus Pulp via Ball Milling and Mercerization, Starch/Staerke, 2018, 180021.

45 L. R. Tang, B. Huang, N. T. Yang, T. Li, Q. L. Lu, W. Y. Lin and X. R. Chen, Organic solvent-free and efficient manufacture of functionalized cellulose nanocrystals via one-pot tandem reactions, Green Chem., 2013, 15(9), 2369-2373.

46 Q. L. Lu, X. Y. Li, L. R. Tang, B. L. Lu and B. Huang, One-pot tandem reactions for the preparation of esterified cellulose nanocrystals with 4-dimethylaminopyridine as a catalyst, RSC Adv., 2015, 5(69), 56198-56204.

47 Y. Habibi, Key advances in the chemical modification of nanocelluloses, Chem. Soc. Rev., 2014, 43(5), 1519-1542.

48 Y. Kojima, J. Minamino, A. Isa, S. Suzuki, H. Ito, R. Makise and M. Okamoto, Binding effect of cellulose nanofibers in wood flour board, J. Wood Sci., 2013, 59(5), 396-401.

49 L. Zhang, T. Tsuzuki and X. Wang, Preparation and characterization on cellulose nanofiber film, Mater. Sci. Forum, 2010, 654-656, 1760-1763.

50 E. Jazaeri, L. Zhang, X. Wang and T. Tsuzuki, Fabrication of carbon nanofiber by pyrolysis of freeze-dried cellulose nanofiber, Cellulose, 2011, 18(6), 1481-1485. 
51 L. Zhang, W. Batchelor, S. Varanasi, T. Tsuzuki and X. Wang, Effect of cellulose nanofiber dimensions on sheet forming through filtration, Cellulose, 2012, 19(2), 561-574.

52 M. Nuruddin, M. Hosur, M. J. Uddin, D. Baah and S. Jeelani, A novel approach for extracting cellulose nanofibers from lignocellulosic biomass by ball milling combined with chemical treatment, J. Appl. Polym. Sci., 2016, 133(9), 42990.

53 V. K. Baheti, R. Abbasi and J. Militky, Ball milling of jute fibre wastes to prepare nanocellulose, World J. Eng., 2012, 9(1), 4550.

54 V. Baheti, V. V. T. Padil, J. Militky, M. Cernik and R. Mishra, Removal of Mercury from Aqueous Environment by Jute Nanofiber, J. Fiber Bioeng. Inf., 2013, 6(2), 175-184.

55 K. T. B. Padal, K. Ramji and V. V. S. Prasad, Isolation and characterization of jute nanofibres, International Journal of Mechanical Engineering and Robotics Research, 2014, 3(2).

56 P. Phanthong, G. Q. Guan, Y. F. Ma, X. G. Hao and A. Abudula, Effect of ball milling on the production of nanocellulose using mild acid hydrolysis method, $J$. Taiwan Inst. Chem. Eng., 2016, 60, 617-622.

57 P. Phanthong, S. Karnjanakom, P. Reubroycharoen, X. G. Hao, A. Abudula and G. Q. Guan, A facile one-step way for extraction of nanocellulose with high yield by ball milling with ionic liquid, Cellulose, 2017, 24(5), 2083-2093.

58 M. M. Zhao, S. Kuga, S. D. Jiang, M. Wu and Y. Huang, Cellulose nanosheets induced by mechanical impacts under hydrophobic environment, Cellulose, 2016, 23(5), 2809-2818.

59 K. Harini, K. Ramya and M. Sukumar, Extraction of nano cellulose fibers from the banana peel and bract for production of acetyl and lauroyl cellulose, Carbohydr. Polym., 2018, 201, 329-339.

60 M. Rajinipriya, M. Nagalakshmaiah, M. Robert and S. Elkoun, Homogenous and transparent nanocellulosic films from carrot, Ind. Crops Prod., 2018, 118, 53-64.

61 P. Huang, M. Wu, S. Kuga, D. Q. Wang, D. Y. Wu and Y. Huang, One-Step Dispersion of Cellulose Nanofibers by Mechanochemical Esterification in an Organic Solvent, Chemsuschem, 2012, 5(12), 2319-2322.

62 P. Huang, M. Wu, S. Kuga and Y. Huang, Aqueous pretreatment for reactive ball milling of cellulose, Cellulose, 2013, 20(4), 2175-2178.

63 P. Huang, Y. Zhao, S. Kuga, M. Wu and Y. Huang, A versatile method for producing functionalized cellulose nanofibers and their application, Nanoscale, 2016, 8(6), 3753-3759.

64 S. Deng, R. Huang, M. Zhou, F. Chen and Q. Fu, Hydrophobic cellulose films with excellent strength and toughness via ball milling activated acylation of microfibrillated cellulose, Carbohydr. Polym., 2016, 154, 129-138.

65 X. Y. Kang, P. P. Sun, S. Kuga, C. Wang, Y. Zhao, M. Wu and Y. Huang, Thin Cellulose Nanofiber from Corncob Cellulose and Its Performance in Transparent Nanopaper, Energy Environ. Sci., 2017, 5(3), 2529-2534.
66 X. M. Rao, S. Kuga, M. Wu and Y. Huang, Influence of solvent polarity on surface-fluorination of cellulose nanofiber by ball milling, Cellulose, 2015, 22(4), 2341-2348.

67 I. Siro and D. Plackett, Microfibrillated cellulose and new nanocomposite materials: a review, Cellulose, 2010, 17(3), 459-494.

68 S. J. Eichhorn, A. Dufresne, M. Aranguren, N. E. Marcovich, J. R. Capadona, S. J. Rowan, C. Weder, W. Thielemans, M. Roman, S. Renneckar, W. Gindl, S. Veigel, J. Keckes, H. Yano, K. Abe, M. Nogi, A. N. Nakagaito, A. Mangalam, J. Simonsen, A. S. Benight, A. Bismarck, L. A. Berglund and T. Peijs, Review: current international research into cellulose nanofibres and nanocomposites, J. Mater. Sci., 2010, 45(1), 1-33.

69 F. Delogu, G. Gorrasi and A. Sorrentino, Fabrication of polymer nanocomposites via ball milling: Present status and future perspectives, Prog. Mater. Sci., 2017, 86, 75-126.

70 S. W. Wang, Q. Feng, J. S. Sun, F. Gao, W. Fan, Z. Zhang, X. H. Li and X. Y. Jiang, Nanocrystalline Cellulose Improves the Biocompatibility and Reduces the Wear Debris of Ultrahigh Molecular Weight Polyethylene via Weak Binding, ACS Nano, 2016, 10(1), 298-306.

71 J. Ambrosio-Martin, A. Lopez-Rubio, M. J. Fabra, G. Gorrasi, R. Pantani and J. M. Lagaron, Assessment of Ball Milling Methodology to Develop Polylactide-Bacterial Cellulose Nanocrystals Nanocomposites, J. Appl. Polym. Sci., 2015, 132(10), 41605.

72 J. Ambrosio-Martin, M. J. Fabra, A. Lopez-Rubio, G. Gorrasi, A. Sorrentino and J. M. Lagaron, Assessment of Ball Milling as a Compounding Technique to Develop Nanocomposites of Poly(3-Hydroxybutyrate-co-3-Hydroxyvalerate) and Bacterial Cellulose Nanowhiskers, J. Polym. Environ., 2016, 24(3), 241-254.

73 E. Abraham, P. A. Elbi, B. Deepa, P. Jyotishkumar, L. A. Pothen, S. S. Narine and S. Thomas, X-ray diffraction and biodegradation analysis of green composites of natural rubber/nanocellulose, Polym. Degrad. Stab., 2012, 97(11), 2378-2387.

74 E. Abraham, B. Deepa, L. A. Pothan, M. John, S. S. Narine, S. Thomas and R. Anandjiwala, Physicomechanical properties of nanocomposites based on cellulose nanofibre and natural rubber latex, Cellulose, 2013, 20(1), 417-427.

75 M. G. Thomas, E. Abraham, P. Jyotishkumar, H. J. Maria, L. A. Pothen and S. Thomas, Nanocelluloses from jute fibers and their nanocomposites with natural rubber: Preparation and characterization, Int. J. Biol. Macromol., 2015, 81, 768-777.

76 S. Deng, J. Ma, Y. Guo, F. Chen and Q. Fu, One-step modification and nanofibrillation of microfibrillated cellulose for simultaneously reinforcing and toughening of poly(E-caprolactone), Compos. Sci. Technol., 2018, 157, 168177. 